\title{
Effects of Serial Passage on the Characteristics and Cardiac and Neural Differentiation of Human Umbilical Cord Wharton's Jelly-Derived Mesenchymal Stem Cells
}

\author{
Jianchun Lian, ${ }^{1,2}$ Shijie Lv, ${ }^{3}$ Chang Liu, ${ }^{1,4}$ Yang Liu, ${ }^{1}$ Shujun Wang, Xin Guo, ${ }^{1}$ Feng Nan, \\ Hua $\mathrm{Yu}^{3}{ }^{3} \mathrm{Xin} \mathrm{He}^{2}{ }^{2}$ Guangwei Sun, ${ }^{1}$ and Xiaojun $\mathrm{Ma}^{1}$ \\ ${ }^{1}$ Laboratory of Biotechnology, Dalian Institute of Chemical Physics, Chinese Academy of Sciences, Dalian 116023, China \\ ${ }^{2}$ The Laboratory Medical College, Dalian Medical University, Dalian 116044, China \\ ${ }^{3}$ Dalian Maternity \& Child Healthcare Hospital, Dalian 116033, China \\ ${ }^{4}$ University of Chinese Academy of Sciences, Beijing 100049, China \\ ${ }^{5}$ Department of Orthopedics, The Second Hospital of Dalian Medical University, Dalian 116023, China
}

Correspondence should be addressed to Yang Liu; liuyang@dicp.ac.cn and Guangwei Sun; sungw@dicp.ac.cn

Received 16 June 2015; Accepted 31 August 2015

Academic Editor: Franca Fagioli

Copyright (C) 2016 Jianchun Lian et al. This is an open access article distributed under the Creative Commons Attribution License, which permits unrestricted use, distribution, and reproduction in any medium, provided the original work is properly cited.

\begin{abstract}
Background and Objective. It is important to guarantee the quality of stem cells. Serial passage is the main approach to expand stem cells. This study evaluated effects of serial passage on the biological characteristics of human umbilical cord Wharton's jellyderived MSCs (WJ MSCs). Methods. Biological properties of WJ MSCs in the early (less than 10 passages, P10), middle (P11-20), and late (more than P20) phases including cell proliferation, cell cycle, phenotype, senescence, oncogene expression, stemness marker expression, and differentiation capacity were evaluated using flow cytometry, real-time PCR, immunocytofluorescence, and western blot. Results. It was found that there were no significant differences in cell proliferation, cell cycle, phenotype, and stemness marker expression in different phases. However, the expression of senescence-related gene, p21, and oncogene, c-Myc, was significantly upregulated in the late phase, which had close relations with the obviously increased cell senescence. Moreover, cardiac differentiation capability of WJ MSCs decreased whereas the propensity for neural differentiation increased significantly in the middle phase. Conclusions. This study reveals that WJ MSCs in the early and middle phases are relatively stable, and effect of serial passage on the lineage-specific differentiation should be considered carefully.
\end{abstract}

\section{Introduction}

Human mesenchymal stem cells (MSCs) have great potential in regenerative medicine, because they can self-renew and differentiate into cellular derivations of three primary germ layers, such as neural cells [1], cardiomyocytes [2], and hepatocytes [3]. MSCs reside in many adult organs or tissues, for example, bone marrow (BM) and adipose, and they also exist in birth-associated tissues, such as umbilical cord (UC), placenta, and amniotic fluid [4]. MSCs were isolated initially from $\mathrm{BM}$, but $\mathrm{BM}$ aspiration is a highly invasive procedure, which is detrimental for patients [5]. Adipose tissue is thought to be a better source than BM because it can be obtained by a less invasive procedure [6]. However, it is reported that the quality of MSCs derived from BM or adipose tissue may decline with progressive age $[7,8]$. Birthassociated tissues were discarded as medical waste before. However, at present, they are gaining popularity as alternative sources of MSCs $[9,10]$. In birth-associated tissues accessed, UC Wharton's jelly-derived MSCs (WJ MSCs) offer the best clinical utility, partly due to their high purity and unique properties [11]. It is reported that WJ MSCs have higher expression of undifferentiated human embryonic stem cell (ESC) markers than BM MSCs, and they also can be induced to be neural progenitors with higher efficiency compared to BM MSCs and adipose-derived MSCs (AD MSCs) $[12,13]$. In 
addition, more and more WJ MSCs are preserved in stem cell banks, which indicates that WJ MSCs have the potential for large-scale applications. Therefore, it appears that WJ MSCs may be an ideal MSC source instead of BM and AD MSCs.

To ensure the accuracy and stability of stem cell research, it is very important to guarantee the quality of cultured stem cells due to the possibility of lost phenotype, malignant transformation, altered lineage-specific differentiation capacity, and so forth during MSC culture in vitro. Serial passage is the main approach to culture MSCs in vitro at present. It has been found that serial passage has different effects on the cellular characteristics of human BM MSCs and AD MSCs [14-16]. However, how serial passage affects the biological characteristics of WJ MSCs is still unclear at present.

Therefore, in this study we investigated effects of serial passage on the specific characteristics of WJ MSCs in the early phase (less than 10 passages, representative passage 7 , P7), middle phase (between 10 and 20 passages, P14), and late phase (more than 20 passages, P21) by concurrently monitoring cell proliferation, cell cycle, phenotype, senescence, oncogene expression, stemness marker expression, differentiation capacity, and so forth. Our results show that there are no significant differences in cell proliferation, cell cycle, phenotype, and stemness marker expression in different phases. However, the expression of senescence-related gene, p21, and oncogene, c-Myc, is significantly upregulated in the late phase, which has close relations with the obviously increased senescence of WJ MSCs. Moreover, cardiac differentiation capability of WJ MSCs might decrease whereas the propensity for neural differentiation might increase significantly in the middle phase. These results indicated that WJ MSCs in the early and middle phases were relatively stable, and the effect of serial passage on the lineage-specific differentiation of $\mathrm{WJ}$ MSCs would be considered carefully in stem cell research, which was important for the quality control of stem cells.

\section{Material and Methods}

2.1. WJ MSC Culture. Primary WJ MSCs from three human UC samples $(n=3)$ were isolated as previously described [12] and then donated by Zhongyuan Union Stem Cell Bioengineering Corporation (Tianjin, China). The cells were cultured in the special WJ MSC culture medium without animal serum that was also kindly gifted by Zhongyuan Union Stem Cell Bioengineering Corporation. The medium was changed every three days and cells were subcultured when reaching $90 \%$ confluence with the seeding density of $10^{4}$ cells $/ \mathrm{cm}^{2}$. The morphology of WJ MSCs was observed under a microscope (Eclipse TE2000-U, Nikon, Tokyo, Japan). Population doubling (PD) and population doubling time (PDT) of the cultured WJ MSCs were calculated according to the previous report [17].

2.2. WJ MSC Proliferation. Cell proliferation was determined by using the cell counting kit-8 (CCK8) (Dojindo Laboratories, Kumamoto, Japan) assay according to the manufacturer's instructions [18]. The absorbance at $450 \mathrm{~nm}$ with a reference wavelength at $630 \mathrm{~nm}$ was recorded using a microplate reader (Well Scan MK3, Labsystems, Dragon, Finland).

2.3. Cell Cycle Analysis. WJ MSCs were harvested and fixed in $70 \%$ cold ethanol overnight. Cell pellets were resuspended in $1 \mathrm{mg} / \mathrm{mL}$ RNase (Sigma-Aldrich, St. Louis, MO, USA) at $37^{\circ} \mathrm{C}$ for $30 \mathrm{~min}$, followed by staining with $50 \mu \mathrm{g} / \mathrm{mL}$ propidium iodide (Sigma-Aldrich). For each sample, $10^{4}$ cells were analyzed by flow cytometry (FACSCalibur, BD, Berkeley, CA, USA). Results were expressed as the percentage of cells in each phase of the cell cycle.

2.4. Apoptosis Analysis. WJ MSCs were treated by using Annexin V-FITC/PI kit (KeyGEN bioTECH, Nanjing, China) according to the manufacturer's instruction. The stained cells were detected with the BD FACSCalibur [19].

2.5. Cell Senescence Analysis. SA- $\beta$-gal activity was detected by the SA- $\beta$-gal staining kit (Biyuntian, Wuhan, China) according to the manufacture's instruction [20]. The percentage of senescent cells was calculated according to 3 staining photographs selected at random in each experiment group.

2.6. Chromosome Analysis. The protocol was based on the previous report with some modifications [21]. In brief, when WJ MSCs (P7, P14, and P21) reached $70-80 \%$ confluence, culture medium was switched to fresh medium containing $0.15 \mu \mathrm{g} / \mathrm{mL}$ colchamine (Sigma-Aldrich) for $3 \mathrm{~h}$. Then cells were dissociated by trypsin-EDTA (Sigma-Aldrich). And cells were treated with the hypotonic solution $(0.75 \mathrm{M} \mathrm{KCl})$ (Sigma-Aldrich) at $37^{\circ} \mathrm{C}$ for $24 \mathrm{~min}$. After that, cells were fixed for $20 \mathrm{~min}$ by using fresh Carnoy's fixative solution, $3: 1$ (v/v) methanol/glacial acetic acid (Sigma-Aldrich). Then cells were dropped from high altitude, air dried, and stained by Giemsa stain (Sigma-Aldrich) for $8 \mathrm{~min}$. Samples were analyzed by chromosome analysis software.

2.7. Flow Cytometric Analysis. WJ MSCs were stained directly with FITC or PE-immunolabeled mouse anti-human monoclonal antibodies against HLA-DR, CD45, CD73, CD90, and CD105 (BD Pharmingen, San Diego, CA, USA). Analysis was performed with the FACSCalibur (BD). Data were expressed as number of cells $/ 10^{6}$ cytometric events.

2.8. Cardiac Differentiation. Cardiac differentiation was performed according to the previous report [22]. Briefly, WJ MSCs were treated with $10 \mu \mathrm{M}$ 5-Aza (Sigma-Aldrich) for $24 \mathrm{~h}$. After that, fresh medium was added for 14 days. Medium was changed every three days and the morphology of differentiated WJ MSCs was observed by using the microscope (Eclipse TE2000-U).

2.9. Neural Differentiation. Neural differentiation of WJ MSCs was performed according to the previous report with some modifications [23]. WJ MSCs were treated with $10 \mathrm{ng} / \mathrm{mL}$ bFGF for $24 \mathrm{~h}$. After that, $1 \%(\mathrm{v} / \mathrm{v})$ DMSO and $100 \mu \mathrm{M} \beta$-hydroxyanisole (BHA, Sigma-Aldrich) were added 
TABLE 1: Real-time PCR primers used in the experiments.

\begin{tabular}{|c|c|c|c|}
\hline Target & Forward primer & Reverse primer & Genbank acc. number \\
\hline p21 & ${\text { 5'-GGACAGCAGAGGAAGACCATGT- } 3^{\prime}}^{\prime}$ & 5'-CGGCGTTTGGAGTGGTAGAA-3' $^{\prime}$ & NM_000389 \\
\hline E-Ras & $5^{\prime}$-GCAAGAGTGCGCTGACCAT-3' & $5^{\prime}$-GCCCAGCACACCATCACA-3' & NM_181532.3 \\
\hline c-Myc & $5^{\prime}$-CCACAGCAAACCTCСТCACA-3' & 5'-CGGTTGTTGCTGATCTGTCTCA- $3^{\prime}$ & NM_002467 \\
\hline Oct3/4 & $5^{\prime}$-GTGGAGGAAGCTGACAACAATGAAA-3' & $5^{\prime}$-GACCGAGGAGTACAGTGCAGTGAAG-3' & NM_002701 \\
\hline Nanog & $5^{\prime}$-CAAAGGCAAACAACCCACTT-3' & $5^{\prime}$-ATTGTTCCAGGTCTGGTTGC-3' & XM_002344636.1 \\
\hline Vimentin & $5^{\prime}$-GGTGGACCAGCTAACCAACGA-3' & $5^{\prime}$-TCAAGGTCAAGACGTGCCAGA-3' & NM_003380.3 \\
\hline Gata4 & 5'-GTTTTTTCCCCTTTGATTTTTGATC-3' & $5^{\prime}$-AACGACGGCAACAACGATAAT- $3^{\prime}$ & NM_002052 \\
\hline Nestin & 5'-GGGTCTACAGAGTCAGATCGCTCA-3' & 5'-AGCGAGAGTTCTCAGCCTCCA-3' & NM_006617 \\
\hline AFP & $5^{\prime}$-TAAACCCTGGTGTTGGCCAG-3' & $5^{\prime}$-ATTTAAACTCCCAAAGCAGCAC-3' & NM_001134 \\
\hline $\mathrm{Nkx} 2.5$ & 5'-CCCCTGGATTTTGCATTCAC-3' & $5^{\prime}$-CGTGCGCAAGAACAAACG-3' & NM_004387 \\
\hline$\beta$-Tubulin III & 5'-CCTATTCAGGCCCGACAACTTTA-3' & 5'-CAGGCAGTCACAATTCTCACACTC-3' & NM_006086 \\
\hline$\beta$-actin & $5^{\prime}$-TGGCACCCAGCACAATGAA-3' & 5'-CTAAGTCATAGTCCGCCTAGAAGCA-3' & NM_001101 \\
\hline
\end{tabular}

for another $24 \mathrm{~h}$. The morphology of differentiated WJ MSCs was also observed.

2.10. Real-Time Polymerase Chain Reaction. Total RNA was extracted using TRIzol reagent (TaKaRa, Shiga, Japan) according to manufacturer's protocol. Reverse transcription was performed with the PrimeScript RT reagent Kit (TaKaRa). PCR amplifications were performed using $2 \mathrm{x}$ SYBR Premix Ex Taq II (TaKaRa). The amplified signals were detected continuously with the Stratagene Mx3000P RealTime Cycler (Agilent Technology, Santa Clara, USA). Primers (listed in Table 1) used in this study were designed by Takara Biotechnology (Dalian, China).

2.11. Immunocytofluorescence. After neural differentiation, cells were fixed in $4 \%$ paraformaldehyde (PFA, SigmaAldrich). Incubation with primary and secondary antibodies was carried out in PBS. For negative controls, the primary antibody was omitted. Nuclear staining was performed with Hoechst 33342 (Sigma-Aldrich). The sources of the antibodies used in this study included the following: anti-Nestin (Sigma-Aldrich), anti- $\beta$-Tubulin III (Sigma-Aldrich), and Alexa Fluor 488 goat anti-rabbit IgG antibody (Invitrogen, Carlsbad, CA, USA). The specimens were viewed with the fluorescence microscope (Eclipse TE2000-U). The percentage of Nestin or $\beta$-Tubulin III positive cells was calculated according to 3 immunostaining photographs selected at random in each experiment group (total cell number more than 300 ).

2.12. Western Blot. Cells were lysed in lysis buffer according to previous report [24] and were centrifuged at $12,000 \mathrm{~g}$ for $20 \mathrm{~min}$, at $4^{\circ} \mathrm{C}$. For each sample, $20 \mu \mathrm{g}$ total protein was electrophoresed through a $10 \%(\mathrm{w} / \mathrm{v})$ acrylamide gel and then transferred to a nitrocellulose membrane (BioRad Laboratories, Hercules, CA, USA). The blots were incubated at $4^{\circ} \mathrm{C}$ overnight with anti- $\alpha$-actinin antibody (SigmaAldrich), and the resulting bands were detected by using diaminobenzidine- (DAB-) based horseradish peroxidase (HRP) reaction. Intensities of the bands were semiquantified using a Gel Logic 2200 PRO imaging system (Carestream Molecular Imaging, New Haven, Connecticut, USA).

2.13. Statistical Analysis. WJ MSCs from three human UC samples were used in this study $(n=3)$. All individual experiments were performed in triplicate. Data were expressed as means \pm standard deviation (SD). One-way ANOVA was performed to test the significance of data. Differences were considered significant at ${ }^{*} P<0.05$ and especially significant at ${ }^{* *} P<0.01$.

\section{Results}

3.1. Morphology, Proliferation, Cell Cycle, Apoptosis, and Senescence of WJ MSCs during Serial Passage. It was found that the morphology of WJ MSCs changed during serial passage. WJ MSCs contained many small raised cells with a fibroblast-like appearance in the early phase (Figure 1(a), P7) while becoming larger and more elongate, and some cells gained an irregular and flat morphology in the middle phase (Figure 1(b), P14). In the late phase, more cells appeared to be irregular and flat, and more inclusions were also found in the cytoplasm (Figure 1(c), P21). In addition, the mean PD of WJ MSCs in the early, middle, and late phases (P7, P14, and P21) was 3, 2.8, and 1.8, and the mean PDT was 24, 34.3, and 66.7 hours, respectively.

CCK8 assay showed that the proliferation rate of WJ MSCs decreased gradually in long-term culture in vitro (Figure 1(d)). Compared to cells in the late phase (P21), there was not a plateau in the growth curve of WJ MSCs in the early and middle phases (P7 and P14) (Figure 1(d)). It was probably due to the very high proliferative ability of P7 and P14 WJ MSCs, which resulted in too high cell density and the rapid decrease of the absorbance after reaching the peak value.

There were no significant differences in cell cycle among all the phases (Figure 1(e)). It was also found that S and G2 phase block phenomenon might exist at higher passages (P14 and P21) compared to P7.

In addition, there were no significant differences in apoptosis among three phases (Figure 1(f)). At different passages, 


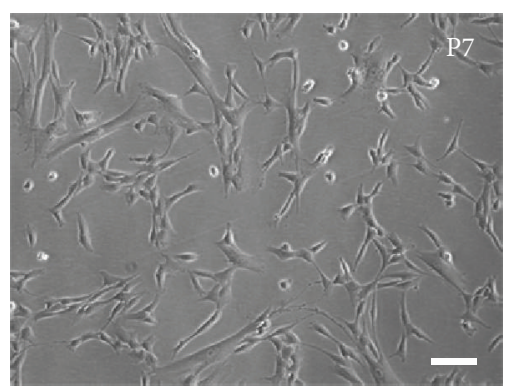

(a)

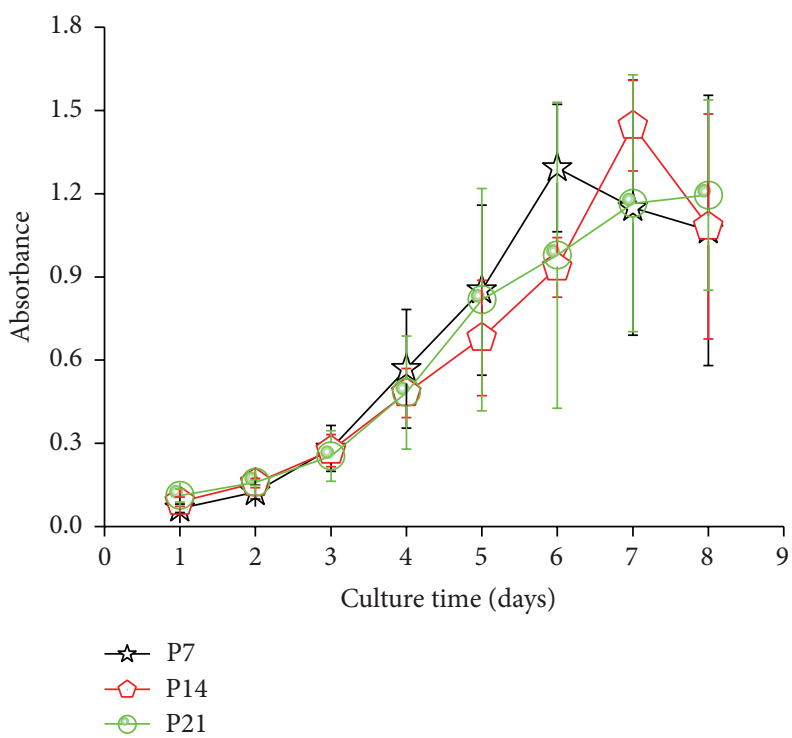

(d)

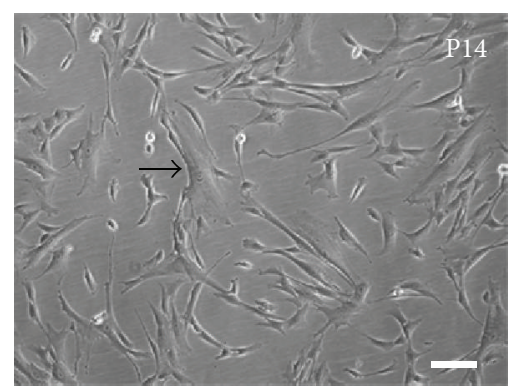

(b)

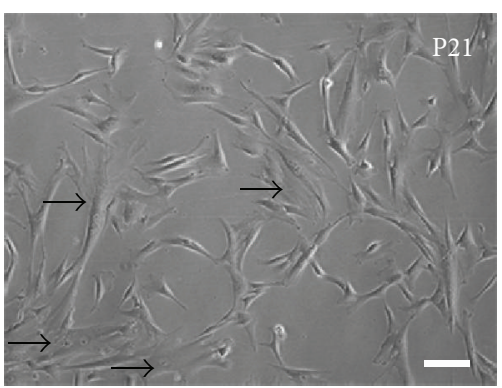

(c)
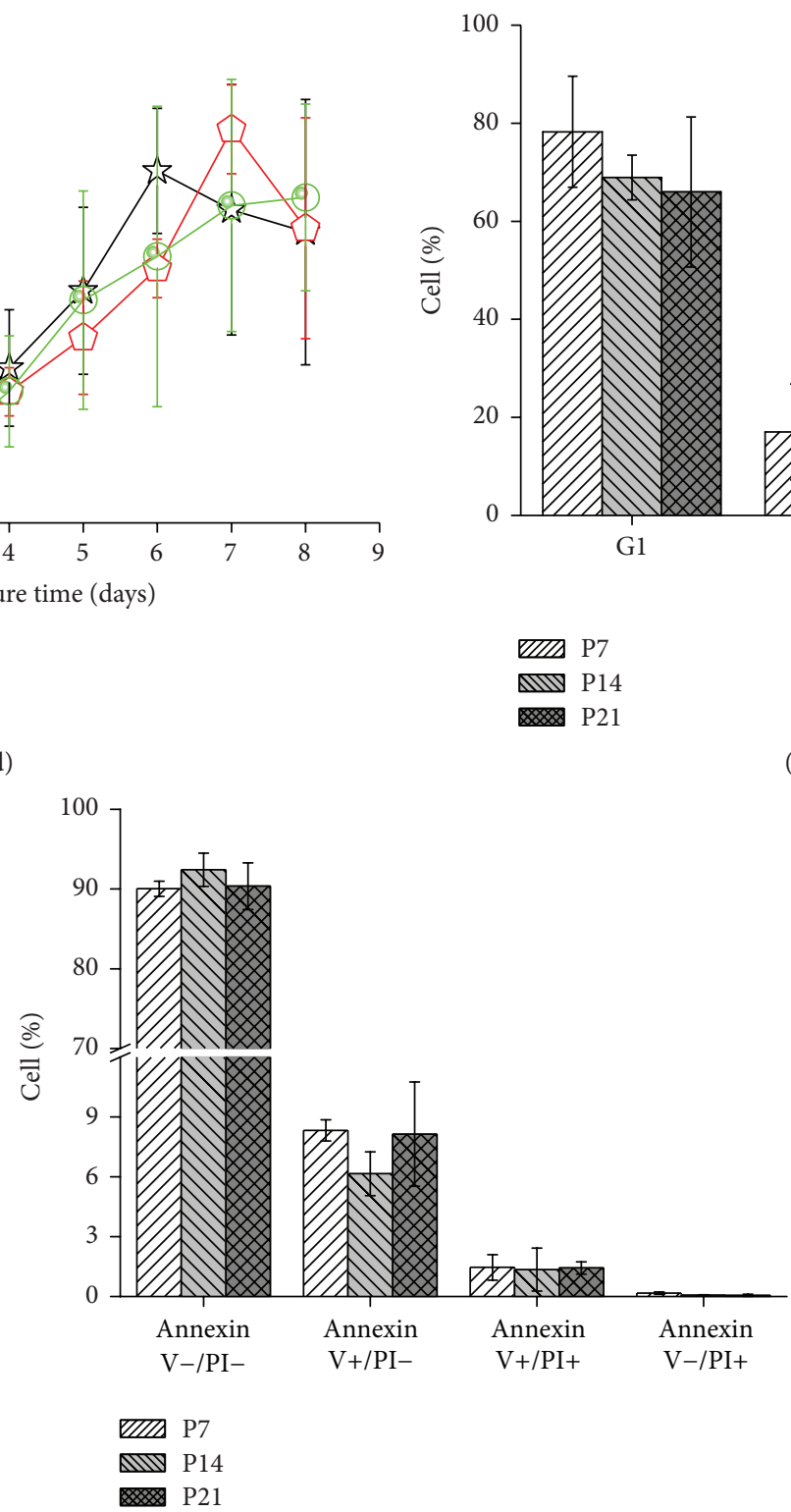

(e)

(f)

FIGURE 1: Morphology, proliferation, cell cycle, and apoptosis of WJ MSCs (Wharton's jelly-derived mesenchymal stem cells) in the early, middle, and late phases. (a) Representative phase-contrast micrographs showed that there were many small raised cells with a fibroblast-like appearance in the early phase, (b) then some cells gained an irregular and flat morphology in the middle phase, and (c) more cells appeared to be irregular and flat in the late phase. Arrows indicated the irregular and flat cells. Bar: $100 \mu \mathrm{m}$. (d) CCK8 (cell counting kit-8) assay showed that the proliferation rate of WJ MSCs decreased gradually in long-term culture in vitro. (e) S and G2 phase block phenomenon might exist at higher passages (P14 and P21) compared to P7. (f) There were no significant differences in apoptosis among three phases. 


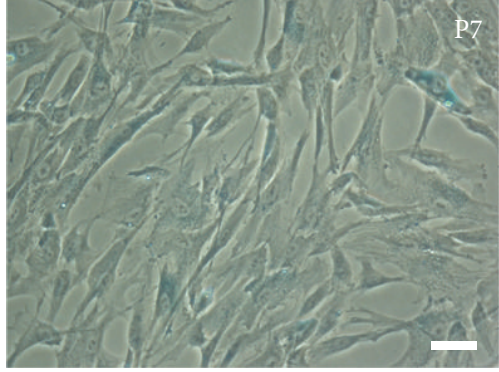

(a)

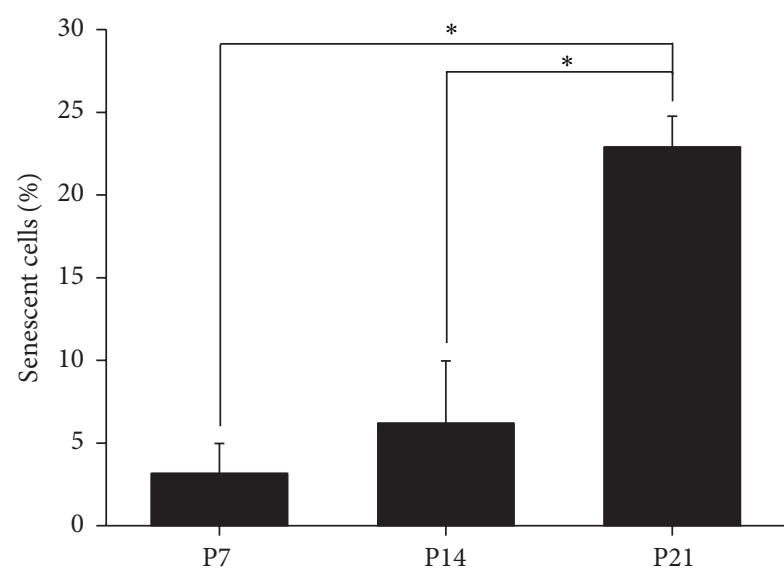

(d)

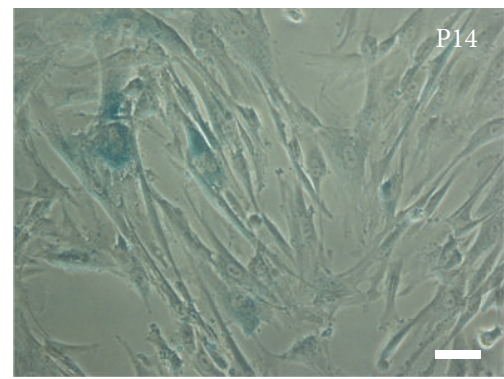

(b)

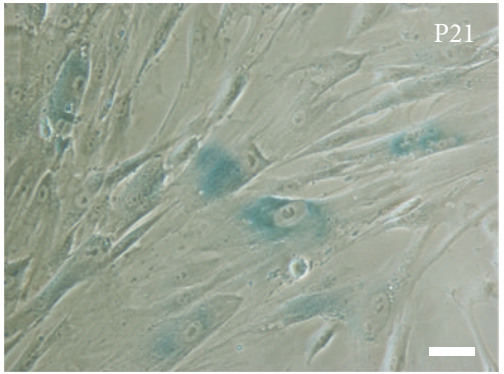

(c)

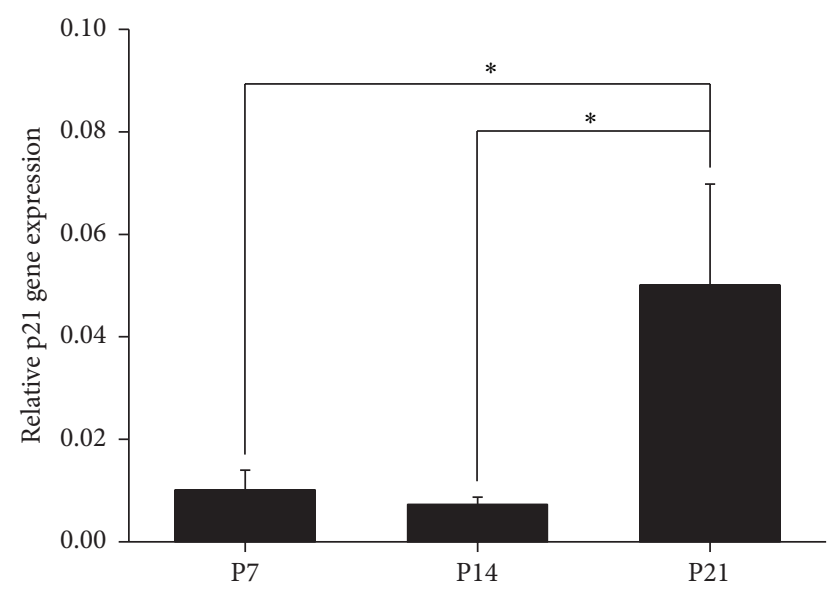

(e)

FIGURE 2: SA- $\beta$-gal staining, percentage of senescent cells, and p21 gene expression in WJ MSCs (Wharton's jelly-derived mesenchymal stem cells) in the early, middle, and late phases. (a) SA- $\beta$-gal staining showed that there were few senescent cells in WJ MSCs in the early phase, (b) senescent cells were found in the middle phase, and (c) more senescent cells appeared in the late phase. Bar: $100 \mu \mathrm{m}$. (d) The percentage of senescent cells in WJ MSCs in the late phase was $22.9 \pm 1.9 \%$, significantly higher than $3.2 \pm 1.8 \%$ in the early phase and $6.2 \pm 3.8 \%$ in the middle phase $\left({ }^{*} \mathrm{P}<0.05\right)$. (e) The gene expression of p21 was upregulated in WJ MSCs in the late phase, which was also significantly higher than that in the early and middle phases $\left({ }^{*} P<0.05\right)$.

the cell percentages of Annexin V-/PI- and Annexin V+/PIwere about $90 \%$ and $9 \%$, respectively, which indicated serial passage did not lead to the significant apoptosis of WJ MSCs.

SA- $\beta$-gal staining showed that there were few senescent cells in WJ MSCs in the early phase (Figure 2(a), P7). With serial passage, senescent cells were found in the middle phase (Figure 2(b), P14), while more senescent cells appeared in the late phase (Figure 2(c), P21). Statistical analysis results of SA$\beta$-gal staining showed that the percentage of senescent cells in P21 WJ MSCs was $22.9 \pm 1.9 \%$, significantly higher than P7 $(3.2 \pm 1.8 \%)$ and P14 $(6.2 \pm 3.8 \%)\left({ }^{*} P<0.05\right)$ (Figure $\left.2(\mathrm{~d})\right)$. In addition, the profile of $\mathrm{p} 21$ gene expression, one of the senescence-related genes [20], was similar to the statistical analysis results of SA- $\beta$-gal staining. The gene expression of p21 was upregulated in WJ MSCs in the late phase, which was also significantly higher than that in the early and middle phases $\left({ }^{*} P<0.05\right)$ (Figure $\left.2(\mathrm{e})\right)$. The above results indicated that serial passage resulted in the senescence of WJ MSCs in the late phase significantly, which might be related to the p21 gene expression.

3.2. Oncogene Expression in WJ MSCs during Serial Passage. Chromosome analysis indicated that serial passage did not change the number of chromosome in WJ MSCs regardless of the phases (Figures 3(a)-3(c)). E-Ras and c-Myc are oncogenes. It was found that there were no significant differences in E-Ras gene expression among different phases, but large individual difference was found in P7 WJ MSCs (Figure 3(d)). However, the c-Myc gene expression in WJ MSCs in the late phase (P21) was upregulated, significantly higher than that in the early phase (P7) and the middle phase $(\mathrm{P} 14)\left({ }^{* *} \mathrm{P}<0.01\right)$ (Figure 3(e)).

3.3. Surface Marker and Pluripotency Gene Expression in WJ MSCs during Serial Passage. It was found that WJ MSCs expressed classic MSC surface markers, including CD73, CD90, and CD105 at high level (about 99\%), and they were negative for hematopoietic markers, CD45 and HLADR (Table 2). Moreover, there were no differences in cell phenotype among different phases, which indicated that serial passage had no significant effect on the classic surface markers of MSCs.

Although surface marker expression was stable during serial passage, the percentage of senescent cells and c-Myc gene expression was significantly increased in WJ MSCs in the late phase compared to the early and middle phases. This 
TABLE 2: Surface marker analysis of WJ MSCs at different passages $(n=3)$.

\begin{tabular}{lccccc}
\hline Passage & HLA-DR & CD45 & CD73 & CD90 & CD105 \\
\hline 7 & $0.59 \pm 0.54 \%$ & $0.57 \pm 0.45 \%$ & $99.92 \pm 0.07 \%$ & $99.98 \pm 0.03 \%$ & $99.74 \pm 0.13 \%$ \\
14 & $0.52 \pm 0.15 \%$ & $0.57 \pm 0.11 \%$ & $99.94 \pm 0.04 \%$ & $99.99 \pm 0.01 \%$ & $99.95 \pm 0.05 \%$ \\
21 & $0.04 \pm 0.06 \%$ & $0.03 \pm 0.04 \%$ & $99.95 \pm 0.03 \%$ & $99.91 \pm 0.1 \%$ & $99.9 \pm 0.09 \%$ \\
\hline
\end{tabular}

P7

P14

P21

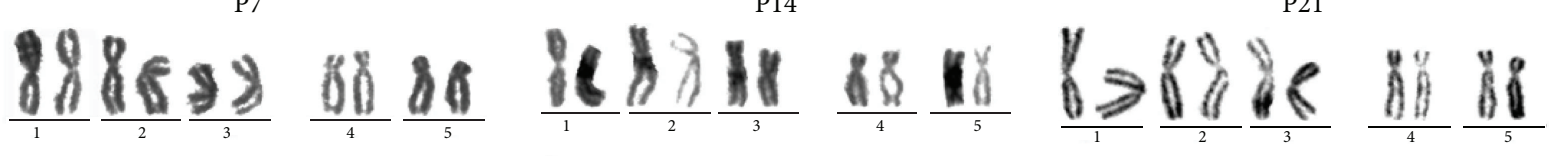

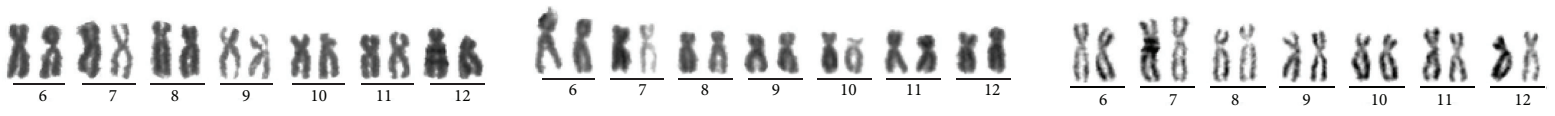

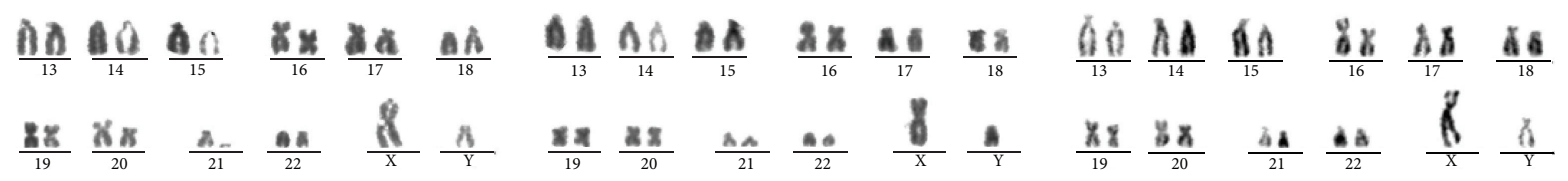

(a)

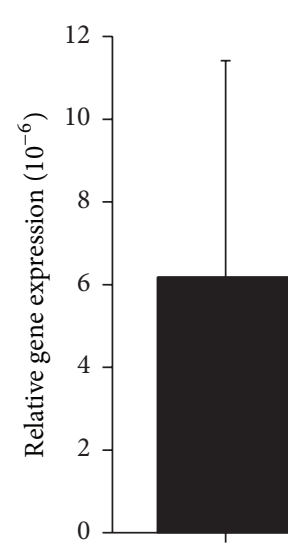

P7
E-Ras

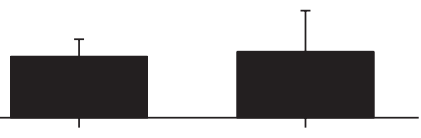

P14 (b)

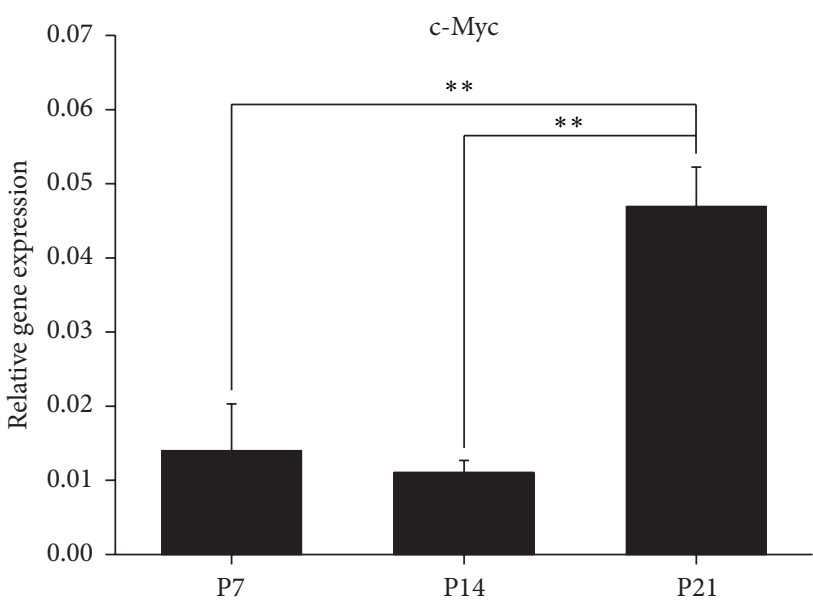

(e)

FIGURE 3: Chromosome analysis and the expression of oncogenes, E-Ras and c-Myc, in WJ MSCs (Wharton's jelly-derived mesenchymal stem cells) in the early, middle, and late phases. (a-c) Chromosome analysis indicated that serial passage did not change the number of chromosome in WJ MSCs regardless of the phases. (d) There were no significant differences in E-Ras gene expression among different phases. (e) The c-Myc gene expression in WJ MSCs in the late phase was upregulated, remarkably higher than the early phase and the middle phase $\left({ }^{* *} P<0.01\right)$.

indicated that WJ MSCs in the early and middle phases might be more stable than cells in the late phase. Therefore, next, we investigated the pluripotency gene expression and committed differentiation of WJ MSCs in the early and middle phases.

WJ MSCs expressed ESC marker genes, Oct3/4 and Nanog, and MSC marker gene, Vimentin. No significant differences in the expression of these genes between P7 and P14 were found, although there was a downward trend (Figures 4(a), 4(b), and 4(c)).

In addition, WJ MSCs also expressed triploblastic progenitor marker genes, for example, mesoderm GATA4, ectoderm Nestin, and entoderm AFP. It was found that there were no significant differences in the expression of these genes between P7 and P14 (Figures 4(d), 4(e), and 4(f)).

3.4. Cardiac Differentiation Capability of WJ MSCs during Serial Passage. WJ MSCs in the early (P7) and middle (P14) phases were treated with 5-Aza for $24 \mathrm{~h}$ to initiate cardiac differentiation. 14 days later, cardiomyocyte-like cells with a stick-like morphology were found in both two groups (induced P7, Figure 5(a)) (induced P14, Figure 5(b)).

The expression of GATA4 and cardiac-specific gene, $\mathrm{Nkx} 2.5$, was upregulated after cardiac induction (Figure 5(c)). The expression of both two genes was slightly 


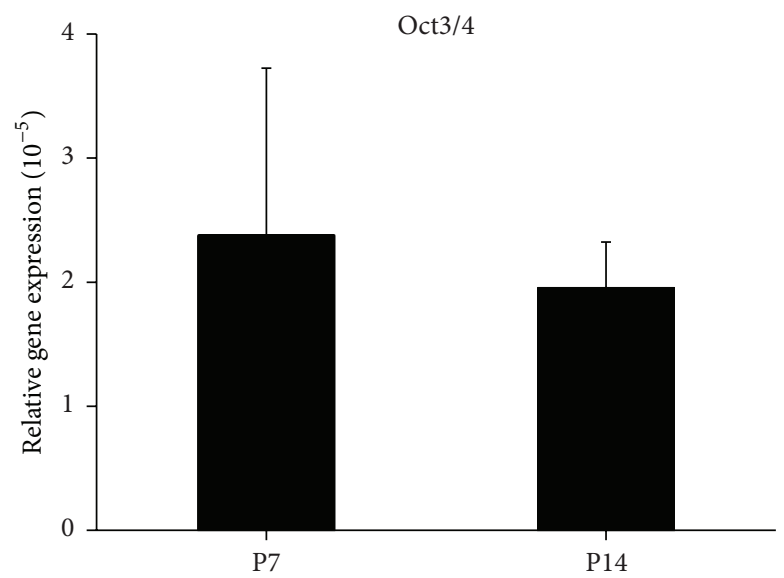

(a)

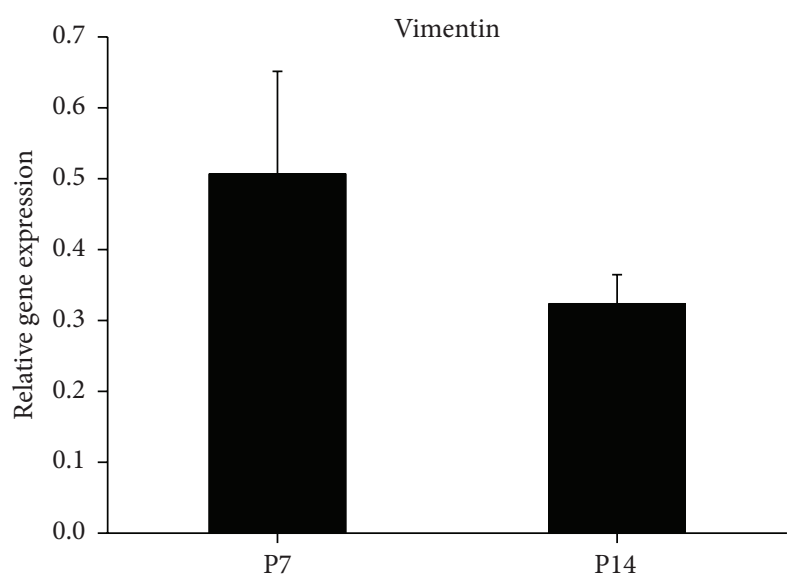

(c)

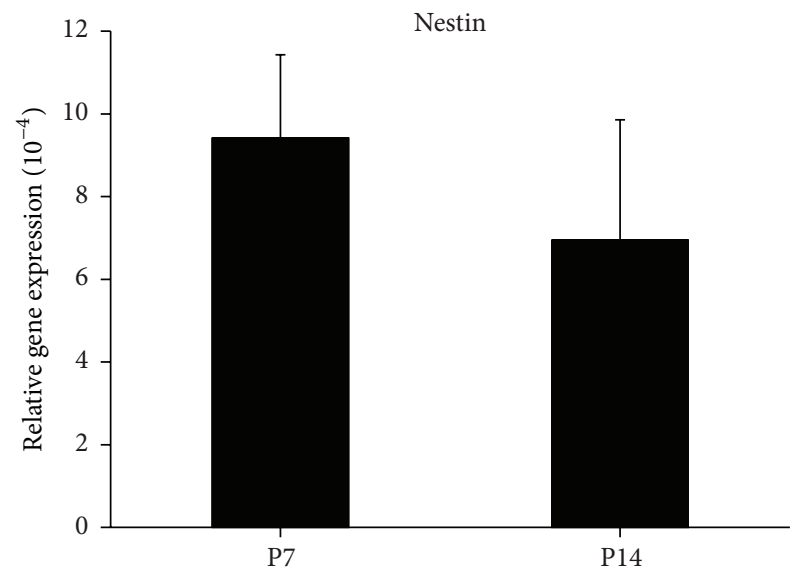

(e)

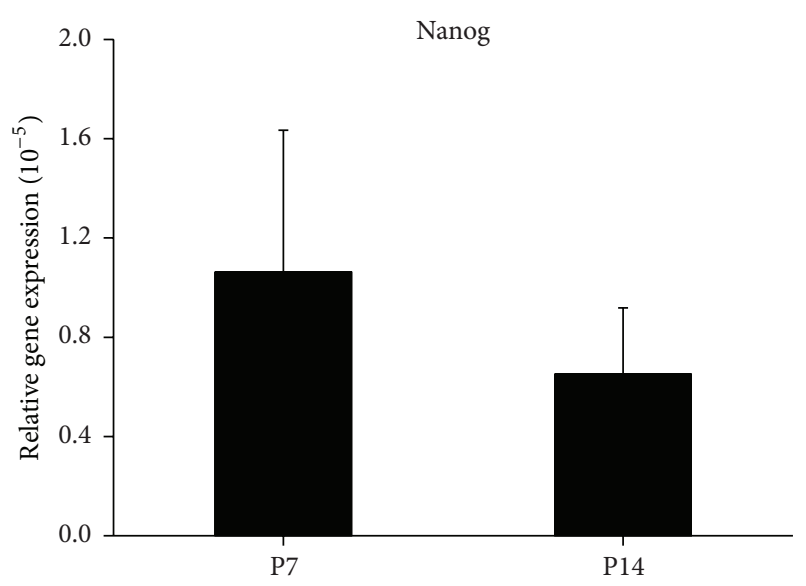

(b)

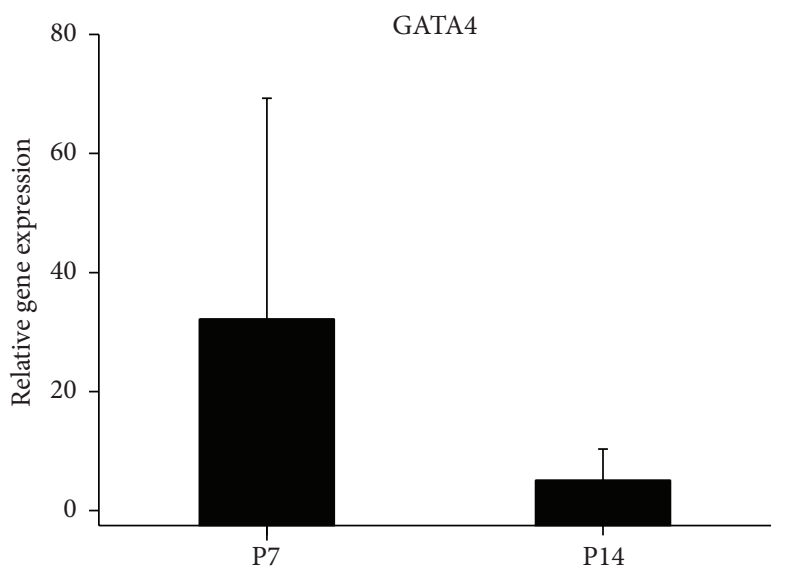

(d)

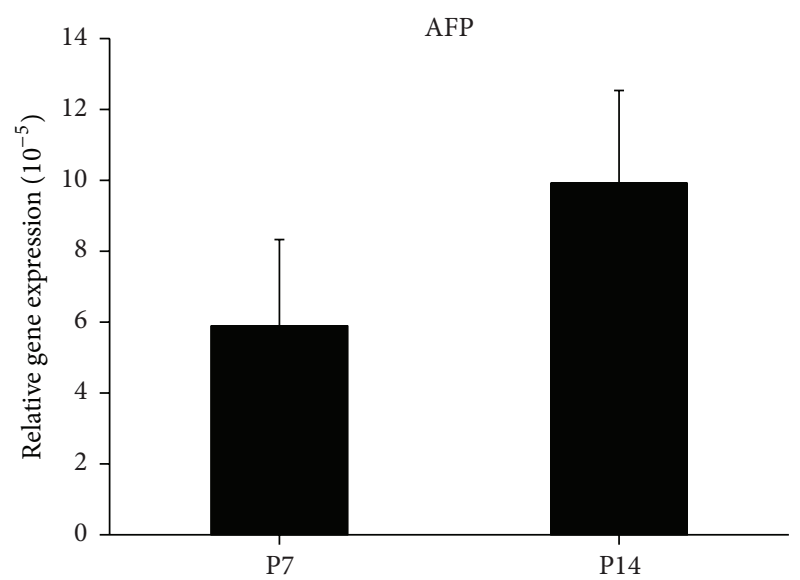

(f)

FIGURE 4: The expression of pluripotency genes and triploblastic progenitor marker genes in WJ MSCs (Wharton's jelly-derived mesenchymal stem cells) in the early and middle phases. $(\mathrm{a}-\mathrm{c})$ There was a downward trend in the expression of Oct3/4, Nanog, and Vimentin in the middle phase compared to the early phase. (d-f) The expression of GATA4, Nestin, and AFP in the early and middle phases.

higher in the group of induced P14 than induced P7 (Figure 5(c)). However, there were no significant differences in gene expression between these two groups.

Moreover, the expression of cardiomyocyte-related protein, $\alpha$-actinin, was also detected by western blot. No $\alpha$ actinin protein expression was detected in P7 and P14
WJ MSCs without induction (P7 control and P14 control) (Figure 5(d)). However, $\alpha$-actinin protein expression was detected in the induced cells (Figure 5(d)). Semiquantified analysis results showed that $\alpha$-actinin protein expression in the group of induced P7 was significantly higher than induced P14 $\left({ }^{*} P<0.05\right)$ (Figure 5(e)). Therefore, serial passage 


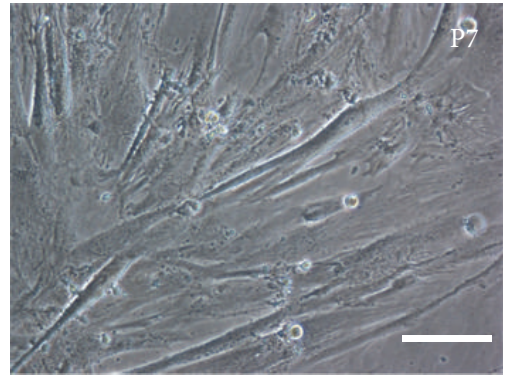

(a)

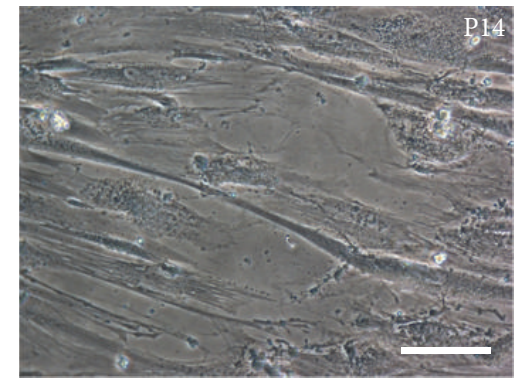

(b)

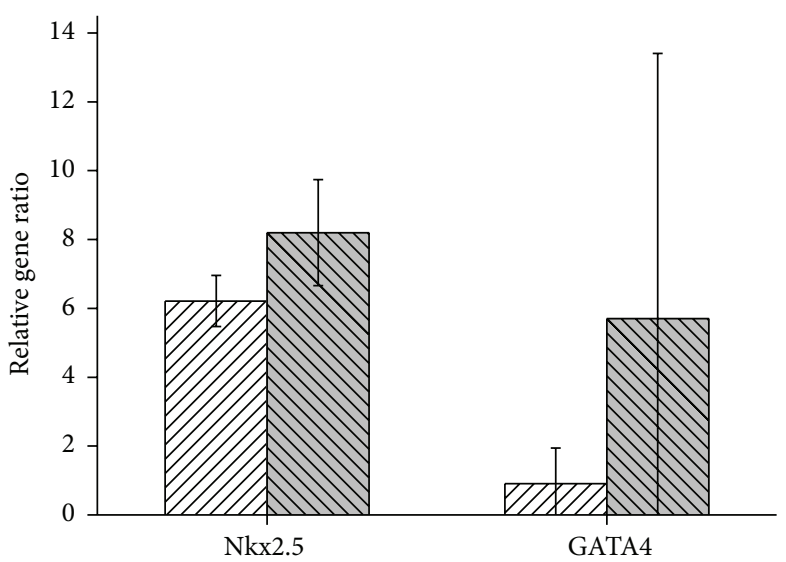

שIIS Induced P7 Induced P14 (c)

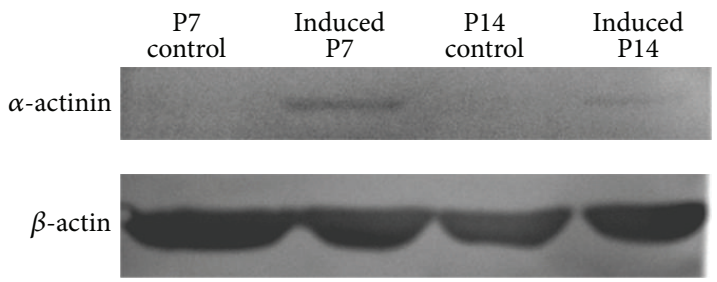

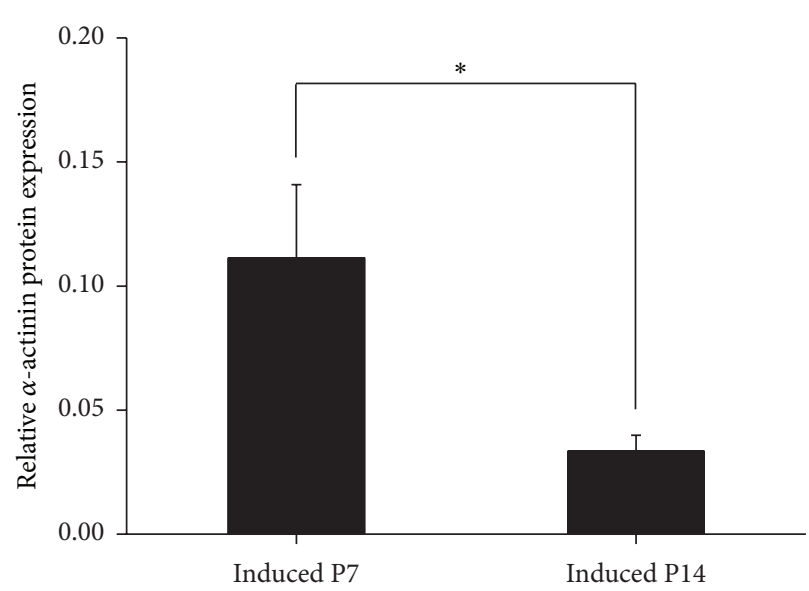

(e)

FIGURE 5: Morphology and expression of cardiac-specific genes and protein in WJ MSCs (Wharton's jelly-derived mesenchymal stem cells) in the early and middle phases after cardiac induction. (a-b) After being treated with 5-Aza for 14 days, cardiomyocyte-like cells with a sticklike morphology were found in induced P7 and P14 cells. Bar: $100 \mu \mathrm{m}$. (c) The gene expression of GATA4 and Nkx2.5 was upregulated after cardiac induction. (d) No $\alpha$-actinin protein expression was detected in normal P7 and P14 WJ MSCs (P7 control and P14 control). However, $\alpha$-actinin protein expression appeared in the induced cells (induced P7 and induced P14). (e) Semiquantified analysis of western blot results showed that $\alpha$-actinin protein expression in the group of induced P7 was significantly higher than induced P14 $\left({ }^{*} P<0.05\right)$, which indicated that serial passage significantly decreased the cardiac differentiation capability of WJ MSCs. 


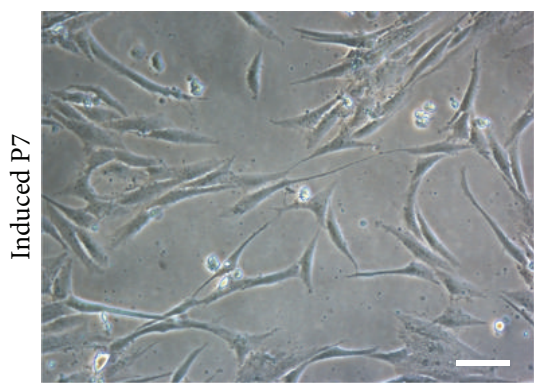

(a)

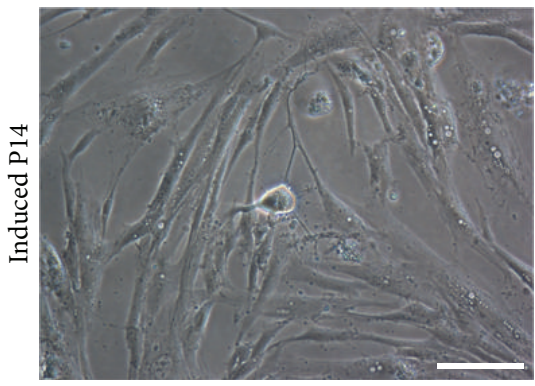

(d)

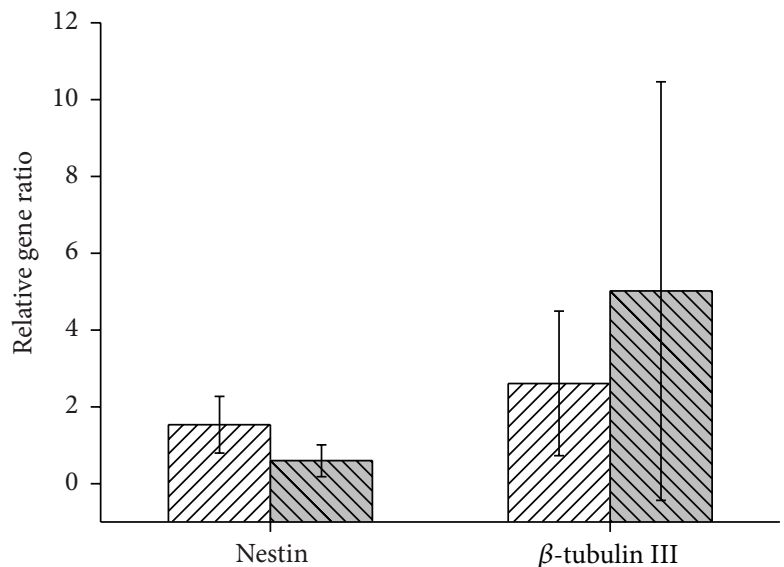

שIn] Induced P7

Induced P14

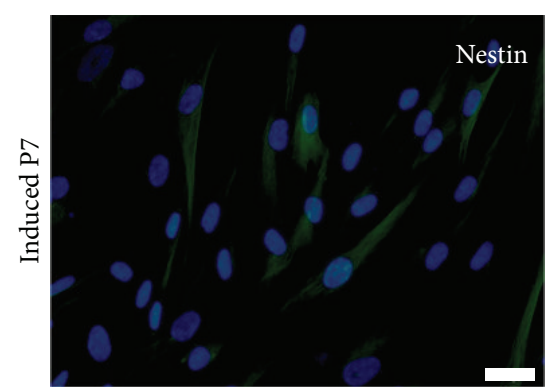

(b)

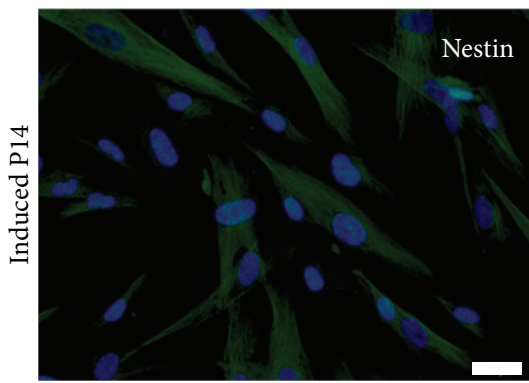

(e)

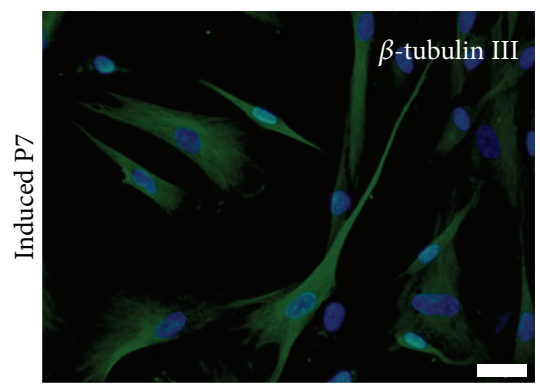

(c)

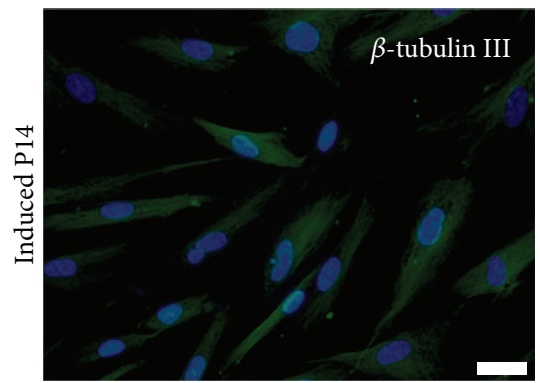

(f)

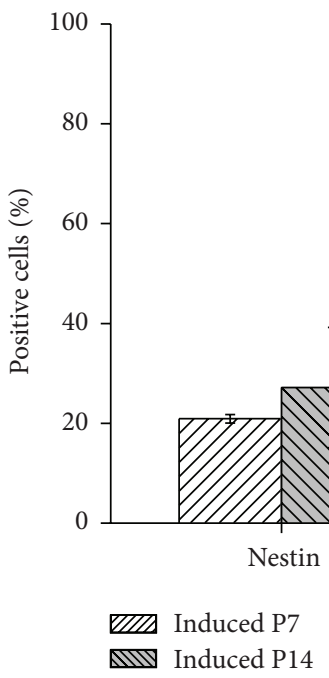

(g)

(h)

FIGURE 6: Morphology and expression of neural-specific genes and protein in WJ MSCs (Wharton's jelly-derived mesenchymal stem cells) in the early and middle phases after neural induction. (a and d) After DMSO and BHA ( $\beta$-hydroxyanisole) treatment, WJ MSCs in the early and middle phases exhibited a neuronal-like morphology. Bar: $100 \mu \mathrm{m}$. (b, c, e, f) Immunocytofluorescence staining results showed that neural induction significantly increased the protein expression of Nestin and $\beta$-Tubulin III in induced cells (induced P7 and induced P14). Bar: $50 \mu \mathrm{m}$. (g) The gene expression of Nestin and $\beta$-Tubulin III was upregulated after neural induction with no significant differences between two groups (induced P7 and induced P14). (h) The percentage of $\beta$-Tubulin III positive cells in the group of P14 induction (70.4 $\pm 4.5 \%$ ) was significantly higher than $\mathrm{P} 7$ induction $(38.6 \pm 10.2 \%)$ according to the statistical analysis results $\left({ }^{*} P<0.05\right)$, which indicated that serial passage significantly increased the neural differentiation capability of WJ MSCs.

significantly decreased the cardiac differentiation capability of WJ MSCs according to protein analysis results, although gene expression had an opposite trend.

3.5. Neural Differentiation Capability of WJ MSCs during Serial Passage. After DMSO and BHA treatment, both WJ
MSCs in the early (P7) and middle (P14) phases exhibited a different morphology (induced P7, Figure 6(a)) (induced P14, Figure 6(d)).

The gene expression of neural progenitor marker, Nestin, and neuronal marker, $\beta$-Tubulin III, was upregulated after neural induction with no significant differences between 
these two groups (induced P7 and induced P14) (Figure 6(g)). And big individual differences in $\beta$-Tubulin III gene expression were found in the group of induced P14 compared to induced P7.

Except gene expression, we also investigated the protein expression of Nestin and $\beta$-Tubulin III in both two groups by immunocytofluorescence staining. Although few WJ MSCs were positive for either Nestin or $\beta$-Tubulin III in the early (P7) and middle (P14) phases (data not shown), neural induction significantly increased the protein expression of these two markers in both two groups (induced P7, Figures 6(b) and 6(c)) (induced P14, Figures 6(e) and 6(f)). Moreover, it was found that the percentage of $\beta$-Tubulin III positive cells in the group of P14 induction $(70.4 \pm 4.5 \%)$ was significantly higher than P7 induction $(38.6 \pm 10.2 \%)$ according to the statistical analysis results $\left({ }^{*} P<0.05\right)$ (Figure $\left.6(\mathrm{~h})\right)$, while no significant differences were found in the percentage of Nestin positive cells between both groups (induced P7, 20.9 \pm $0.8 \%$, and induced P14, $27.2 \pm 12 \%$ ). Therefore, these results indicated that serial passage significantly increased the neural differentiation capability of WJ MSCs.

\section{Discussion}

During serial passage, we found that the senescence of $\mathrm{WJ}$ MSCs was increased significantly in the late phase $(22.9 \pm$ $1.9 \%)$, compared to the middle phase $(6.2 \pm 3.8 \%)$ and the early phase $(3.2 \pm 1.8 \%)\left({ }^{*} P<0.05\right)$ (Figure $\left.2(\mathrm{~d})\right)$. Cheng et al. investigated the senescence of whole UC tissue-derived MSCs in the early phase (P2-10), the middle phase (P11-20), and the late phase (more than P20), and they found that the percentage of senescent cells was increased significantly in the late phase $(76 \%)$, compared to the middle phase $(28 \%)$ and the early phase (very few) [20], which had the similar trend to our results. However, the percentage of senescent cells either in the middle (P14) or late phase (P21) was much lower in this study compared to their results. On one hand, the difference in cell properties between WJ MSCs and whole UC tissue-derived MSCs might lead to this distinction. Except Wharton's jelly, whole UC tissue also contains other compartments, including amnion, subamnion, perivascular region, and umbilical blood vessel endothelium [11]. Several types of stem cell populations with varied stemness properties exist in these compartments [25]. So whole UC tissue-derived MSCs contain mixed stem cell populations with varied properties [11], which may result in the difference in senescence between WJ and whole UC tissue-derived MSCs. On the other hand, MSC culture medium might also have relations with the senescence. Cheng et al. used DF-12 medium containing 10\% FBS while WJ MSCs were cultured in special culture medium without animal serum gifted by a company in this study. This special culture medium is developed for stem cell therapy in the future. Although the recipe of the special culture medium was unknown, it seemed that this optimized culture medium is significantly beneficial to reduce the senescence of WJ MSCs during long-term culture.

It is reported that p 21 gene, one of the senescent-related genes, is important for whole UC tissue-derived MSCs [20,
26]. And the increased c-Myc gene expression in AD MSCs is also relevant to the senescence during serial passage [27]. In this work, we found that both p21 and c-Myc gene expression in WJ MSCs were increased significantly in the late phase $\left({ }^{*} P<0.05\right.$, Figure $\left.2(\mathrm{e})\right)\left({ }^{* *} P<0.01\right.$, Figure 3(e)), which showed the same trend as the percentage of senescent cells $\left({ }^{*} P<0.05\right.$, Figure $\left.2(\mathrm{~d})\right)$. Therefore, our results indicated that both p21 and c-Myc gene expression had close relations with the senescence of WJ MSCs during long-term culture in vitro, and these two genes may be used as markers for senescence monitoring.

Except the senescence, the upregulated c-Myc gene expression is also associated with in vitro spontaneous transformation of human MSCs [27]. Sawada et al. found that the c-Myc gene expression in human MSCs from some donors could be significantly upregulated in long-term culture, accompanied with the increased percentage of c-Myc aberrant cells [28], which indicated that long-term culture might increase the safety risk of MSCs and c-Myc expression was useful for the early evaluation. In this study, we found that cMyc gene expression was significantly increased in WJ MSCs in the late phase $\left({ }^{* *} P<0.01\right.$, Figure $\left.3(\mathrm{e})\right)$, which showed that the safety risk of WJ MSCs might be increased significantly in the late phase. Next, it is well worth evaluating the in vivo tumorigenicity of WJ MSCs with higher c-Myc gene expression after serial passage.

In this study, the cardiac differentiation capability of WJ MSCs decreased $\left({ }^{*} P<0.05\right.$ ) (Figure 5(e)) whereas neural differentiation capability increased significantly $\left({ }^{*} P<\right.$ 0.05) (Figure 6(h)) in the middle phase compared to the early phase. In addition, for whole UC tissue-derived MSCs, adipogenic differentiation decreases while osteogenic differentiation starts to dominate during serial passage [20]. These results indicated that the effect of serial passage might be relevant to the lineage-specific differentiation of WJ MSCs.

However, it was also found that the SD of the gene expression data, such as GATA4 (Figure 5(c)) and $\beta$-Tubulin III (Figure 6(g)), was larger between different UC samples after cardiac or neural induction, which was probably due to the individual difference. The properties of fetal stem cells are reported to have relations with pregnancy [29]. This indicates that, next, more UC samples should be investigated, and some clinical indexes, including volume and weight of UC or placenta of the puerpera, weight and age of the puerperal, and weight and development of the neonate, should be recorded carefully. By statistical analysis, we might find the possible relationship between stem cell properties and clinical indexes, which is very helpful to obtain better UC samples containing WJ MSCs with higher quality and stability. Although it was found that there were significant differences in $\alpha$-actinin protein expression or $\beta$-Tubulin III positive cells after cardiac or neural differentiation between P7 and P14 in this study, however, it is necessary to check more markers and perform functional assays to further evaluate stem cell differentiation [30].

In addition, there are some different variation trends among MSCs from different sources during serial passage, such as surface marker expression and differentiation capacity. For AD MSCs, CD105 expression declines during serial 
passage [15]. However, for BM and WJ MSCs, the expression of CD105 and CD73 is maintained at high level during longterm culture [16, 31] (Table 2). Moreover, long-term culture does not decrease the neuronal differentiation capability of BM MSCs [16] while neural differentiation capability increased significantly in the middle phase compared to the early phase in this study (Figure 6(h)). These results indicate that effects of serial passage on MSC properties are also dependent on MSC sources.

\section{Conclusion}

We found that the expression of senescence-related gene, p21, and oncogene, c-Myc, was significantly upregulated in the late phase, which had close relations with the obviously increased senescence of WJ MSCs. And cardiac differentiation capability of WJ MSCs decreased whereas the propensity for neural differentiation increased significantly in the middle phase compared to the early phase. These results indicated that WJ MSCs in the early and middle phases were relatively stable, and the effect of serial passage on the lineage-specific differentiation of WJ MSCs would be considered carefully in stem cell research. Therefore, it is necessary to monitor the quality of WJ MSCs during culture in vitro, which would be helpful to choose relatively stable stem cells according to specific purposes, maximize the potential of stem cells, and improve the reproducibility of strategies and biotechnologies developed based on stem cells.

\section{Conflict of Interests}

The authors declare that there is no conflict of interests regarding the publication of this paper.

\section{Acknowledgments}

The authors would like to acknowledge the generous giving of WJ MSCs and special cell culture medium from Zhongyuan Union Stem Cell Bioengineering Corporation (Tianjin, China). This work was supported by National Natural Science Foundation of China (no. 31271055 and no. 31470944) and Dalian Science and Technology Foundation (2012J21DW029 and 2009J22DW029).

\section{References}

[1] T. K. Ng, V. R. Fortino, D. Pelaez, and H. S. Cheung, "Progress of mesenchymal stem cell therapy for neural and retinal diseases," World Journal of Stem Cells, vol. 6, no. 2, pp. 111-119, 2014.

[2] A. Supokawej, P. Kheolamai, K. Nartprayut et al., "Cardiogenic and myogenic gene expression in mesenchymal stem cells after 5-azacytidine treatment," Turkish Journal of Hematology, vol. 30, no. 2, pp. 115-121, 2013.

[3] X. B. Wu and R. Tao, "Hepatocyte differentiation of mesenchymal stem cells," Hepatobiliary \& Pancreatic Diseases International, vol. 11, no. 4, pp. 360-371, 2012.

[4] B. R. Sousa, R. C. Parreira, E. A. Fonseca et al., "Human adult stem cells from diverse origins: an overview from multiparametric immunophenotyping to clinical applications," Cytometry Part A, vol. 85, no. 1, pp. 43-77, 2014.
[5] M. F. Pittenger, A. M. Mackay, S. C. Beck et al., "Multilineage potential of adult human mesenchymal stem cells," Science, vol. 284, no. 5411, pp. 143-147, 1999.

[6] N. A. Kia, A. R. Bahrami, M. Ebrahimi et al., "Comparative analysis of chemokine receptor's expression in mesenchymal stem cells derived from human bone marrow and adipose tissue," Journal of Molecular Neuroscience, vol. 44, no. 3, pp. 178185, 2011.

[7] A. I. Caplan, "Why are MSCs therapeutic? New data: new insight," The Journal of Pathology, vol. 217, no. 2, pp. 318-324, 2009.

[8] E. Fossett, W. S. Khan, U. G. Longo, and P. J. Smitham, "Effect of age and gender on cell proliferation and cell surface characterization of synovial fat pad derived mesenchymal stem cells," Journal of Orthopaedic Research, vol. 30, no. 7, pp. 10131018, 2012.

[9] T. Nagamura-Inoue and H. He, "Umbilical cord-derived mesenchymal stem cells: their advantages and potential clinical utility," World Journal of Stem Cells, vol. 6, no. 2, pp. 195-202, 2014.

[10] J. Wang, Z. Zhu, Y. Huang et al., “The subtype CD200-positive, chorionic mesenchymal stem cells from the placenta promote regeneration of human hepatocytes," Biotechnology Letters, vol. 36, no. 6, pp. 1335-1341, 2014.

[11] A. Bongso and C. Y. Fong, "The therapeutic potential, challenges and future clinical directions of stem cells from the Wharton's jelly of the human umbilical cord," Stem Cell Reviews and Reports, vol. 9, no. 2, pp. 226-240, 2013.

[12] U. Nekanti, V. B. Rao, A. G. Bahirvani, M. Jan, S. Totey, and M. Ta, "Long-term expansion and pluripotent marker array analysis of Wharton's jelly-derived mesenchymal stem cells," Stem Cells and Development, vol. 19, no. 1, pp. 117-130, 2010.

[13] S. Balasubramanian, C. Thej, P. Venugopal et al., "Higher propensity of Wharton's jelly derived mesenchymal stromal cells towards neuronal lineage in comparison to those derived from adipose and bone marrow," Cell Biology International, vol. 37, no. 5, pp. 507-515, 2013.

[14] S. Kawauchi, H. Terasaki, M. Katano et al., "Quality control and monitoring for the isolation process of mesenchymal stem cells and their differentiation into osteoblasts," Genetic Testing and Molecular Biomarkers, vol. 14, no. 2, pp. 269-282, 2010.

[15] M. E. Wall, S. H. Bernacki, and E. G. Loboa, "Effects of serial passaging on the adipogenic and osteogenic differentiation potential of adipose-derived human mesenchymal stem cells," Tissue Engineering, vol. 13, no. 6, pp. 1291-1298, 2007.

[16] M. L. M. Khoo, B. Shen, H. Tao, and D. D. F. Ma, "Longterm serial passage and neuronal differentiation capability of human bone marrow mesenchymal stem cells," Stem Cells and Development, vol. 17, no. 5, pp. 883-896, 2008.

[17] M. M. Nur Fariha, K. H. Chua, G. C. Tan, A. E. Tan, and A. R. Hayati, "Human chorion-derived stem cells: changes in stem cell properties during serial passage," Cytotherapy, vol. 13, no. 5, pp. 582-593, 2011.

[18] Y. Liu, C. Liu, F. Nan et al., "Microcapsule co-culture system enhances neural differentiation of mesenchymal stem cells," Journal of Hard Tissue Biology, vol. 22, no. 2, pp. 241-248, 2013.

[19] B. Tang, Y. Zhang, R. Liang et al., "Activation of the $\delta$ opioid receptor inhibits serum deprivation-induced apoptosis of human liver cells via the activation of PKC and the mitochondrial pathway," International Journal of Molecular Medicine, vol. 28, no. 6, pp. 1077-1085, 2011. 
[20] H. Cheng, L. Qiu, J. Ma et al., "Replicative senescence of human bone marrow and umbilical cord derived mesenchymal stem cells and their differentiation to adipocytes and osteoblasts," Molecular Biology Reports, vol. 38, no. 8, pp. 5161-5168, 2011.

[21] D. Moralli, M. Yusuf, M. A. Mandegar, S. Khoja, Z. L. Monaco, and E. V. Volpi, "An improved technique for chromosomal analysis of human ES and iPS cells," Stem Cell Reviews and Reports, vol. 7, no. 2, pp. 471-477, 2011.

[22] Q. Qian, H. Qian, X. Zhang et al., "5-Azacytidine induces cardiac differentiation of human umbilical cord-derived mesenchymal stem cells by activating extracellular regulated kinase," Stem Cells and Development, vol. 21, no. 1, pp. 67-75, 2012.

[23] K. Mareschi, M. Novara, D. Rustichelli et al., "Neural differentiation of human mesenchymal stem cells: evidence for expression of neural markers and eag $\mathrm{K}^{+}$channel types," Experimental Hematology, vol. 34, no. 11, pp. 1563-1572, 2006.

[24] B. Tang, J. Du, J. Wang et al., "Alpinetin suppresses proliferation of human hepatoma cells by the activation of MKK7 and elevates sensitization to cis-diammined dichloridoplatium," Oncology Reports, vol. 27, no. 4, pp. 1090-1096, 2012.

[25] M. T. Conconi, R. D. Liddo, M. Tommasini, C. Calore, and P. P. Parnigotto, "Phenotype and differentiation potential of stromal populations obtained from various zones of human umbilical cord: an overview," Open Tissue Engineering and Regenerative Medicine Journal, vol. 4, no. 1, pp. 6-20, 2011.

[26] U. Herbig, W. A. Jobling, B. P. C. Chen, D. J. Chen, and J. M. Sedivy, "Telomere shortening triggers senescence of human cells through a pathway involving ATM, p53, and p21 ${ }^{\mathrm{CIP} 1}$, but

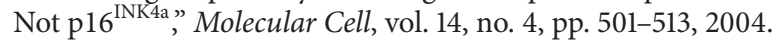

[27] D. Rubio, S. Garcia, M. F. Paz et al., "Molecular characterization of spontaneous mesenchymal stem cell transformation," PLoS ONE, vol. 3, no. 1, Article ID e1398, 2008.

[28] R. Sawada, A. Matsuoka, Y. Matsuda, and T. Tsuchiya, "Change in characteristics of human mesenchymal stem cells during the in vitro culture-c-myc Gene expression and chromosome aberrations at the c-myc locus," Yakugaku Zasshi, vol. 128, no. 12, pp. 1851-1856, 2008.

[29] M. Izumi, B. J. Pazin, C. F. Minervini et al., "Quantitative comparison of stem cell marker-positive cells in fetal and term human amnion," Journal of Reproductive Immunology, vol. 81, no. 1, pp. 39-43, 2009.

[30] S. P. Sheehy, F. Pasqualini, A. Grosberg, S. J. Park, Y. AratynSchaus, and K. K. Parker, "Quality metrics for stem cell-derived cardiac myocytes," Stem Cell Reports, vol. 2, no. 3, pp. 282-294, 2014.

[31] J. Lo Surdo and S. R. Bauer, "Quantitative approaches to detect donor and passage differences in adipogenic potential and clonogenicity in human bone marrow-derived mesenchymal stem cells," Tissue Engineering Part C: Methods, vol. 18, no. 11, pp. 877-889, 2012. 

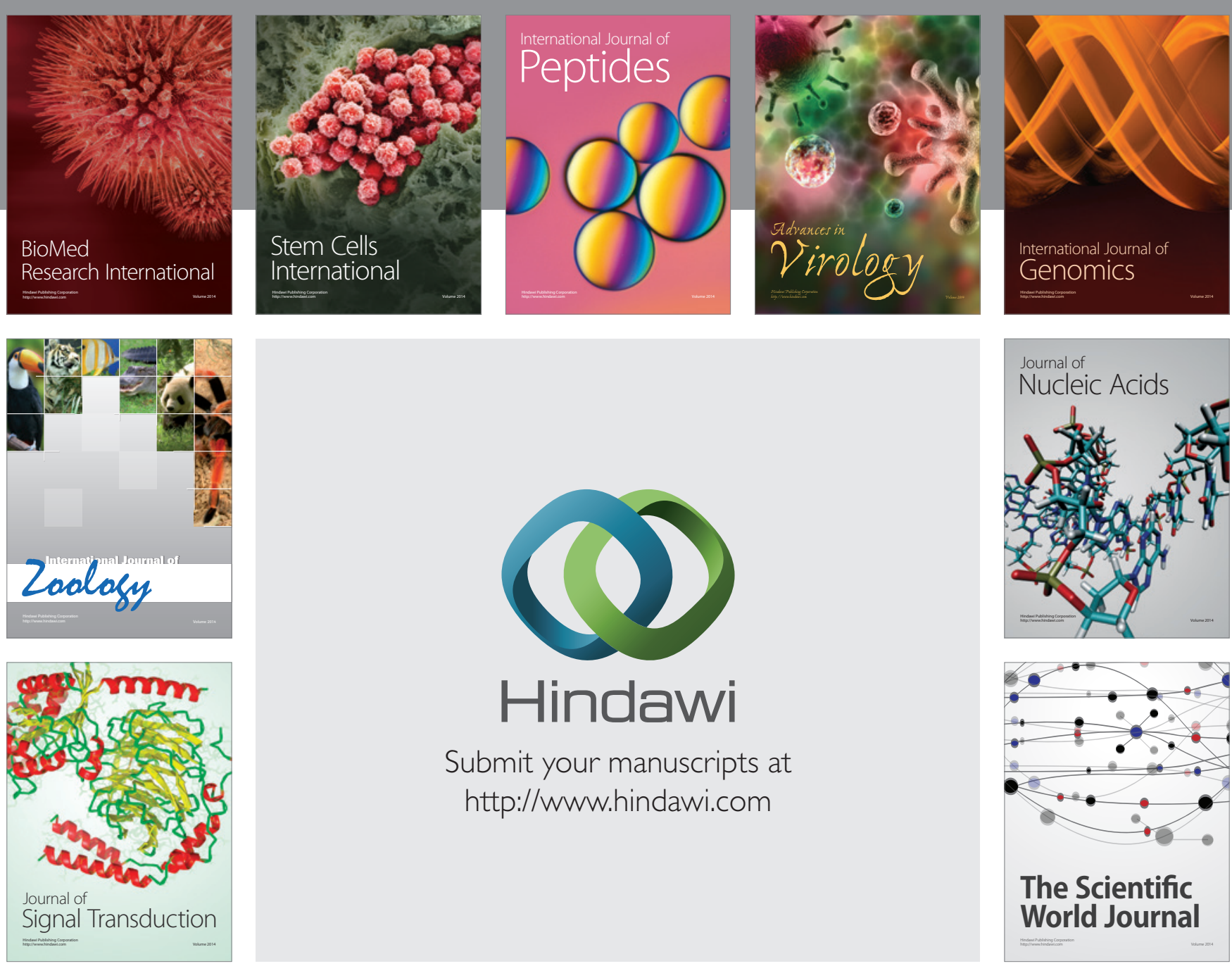

Submit your manuscripts at

http://www.hindawi.com
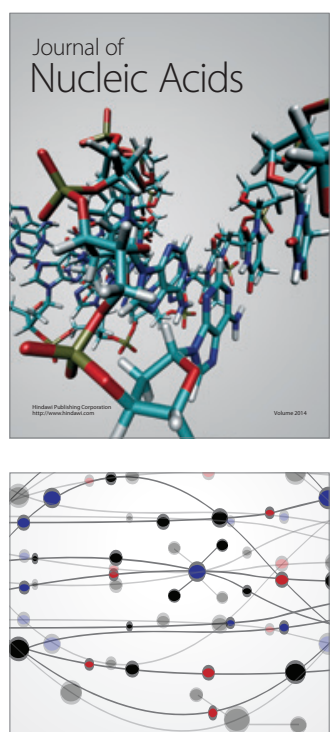

The Scientific World Journal
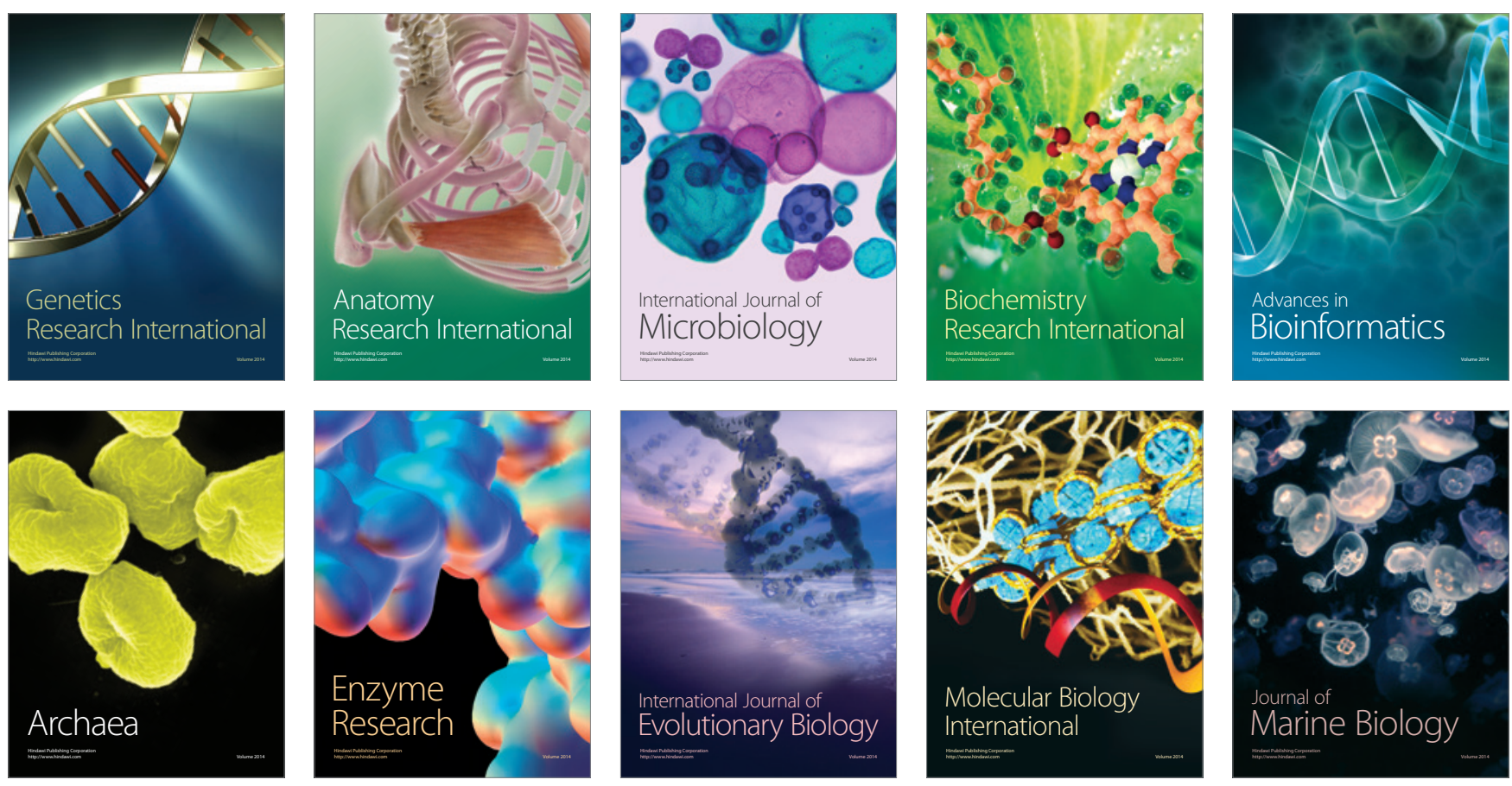